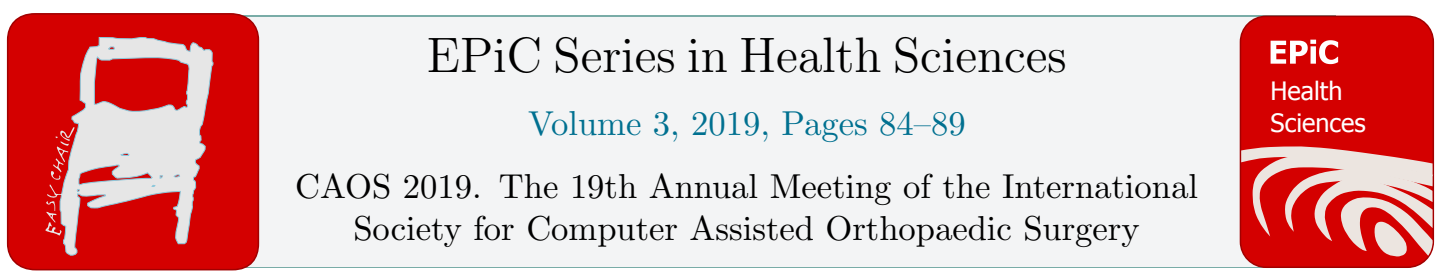

\title{
A Retrospective Comparison of Functional Outcomes of Robotic-Assisted and Conventional Unicompartmental Knee Arthroplasty
}

\author{
Meredith P Crizer, BS; Andrew Battenberg, MD; Mikayla E McGrath, BS; Seong Jin Kim, MPH; \\ and Jess H Lonner, MD \\ Rothman Orthopedic Institute \\ Jess.Lonner@rothmanortho.com
}

\begin{abstract}
INTRODUCTION: Unicompartmental knee arthroplasty (UKA) is effective for alleviating symptoms of arthritis in a single knee compartment; however, errors in alignment and instability may predispose to failure. Robotic technology has improved precision, but its impact on functional recovery after UKA remains unknown. The purpose of this study was to compare early functional recovery, pain, and radiographic alignment in UKA performed with either robotic assistance or conventional methods.

METHODS: All consecutive patients undergoing UKA by a single physician from January 2015 to March 2018 were retrospectively reviewed. Lower Extremity Functional Scale (LEFS), KOOS Jr, and VAS (0-10) outcomes scores were collected preoperatively, 1, 6, and 12 weeks postoperatively. Radiographic alignment was assessed at the initial postoperative visit.

RESULTS: There were 161 patients, 65 conventional and 96 robotic. At baseline, there was no difference in average age, BMI, or outcomes scores between the two groups. Average preoperative pain was significantly higher in conventional patients ( 6.1 vs. $5.4 ; \mathrm{p}=0.04)$. At 3 weeks post-op, conventional UKA patients still had significantly higher pain levels ( 3.9 vs. $3.1 ; \mathrm{p}=.02)$. Both groups showed significant improvement in LEF $(p<.0001)$ scores over time $(p<.0001)$. Significant improvement in KOOS Jr. scores from pre-op (52.3) to 6 and 12-weeks postoperatively $(67.6,69.8$; $\mathrm{p}<0.001)$. When comparing PCS of the VR/SF-12, the robotic-assisted group had significantly higher improvement compared to the conventional group at 6 -weeks ( 40.6 vs. $35.6 ; \mathrm{p}=0.02$ ).

CONCLUSION: Robotic assisted UKA resulted in fewer radiographic outliers, and more rapid recovery with less early postoperative pain although functional differences tended to equilibrate by 3 months postoperatively.
\end{abstract}




\section{Introduction}

Unicompartmental Knee Arthroplasty (UKA) has been proven to alleviate symptoms of isolated arthritis in a single knee compartment [1-3]. The use of robotics in these cases has become of great interest as it aims to reduce human error by improving precision and accuracy of implant positioning [4]. These factors are particularly important for patients undergoing UKA, where studies have shown that small errors in alignment may predispose patients to mechanical failure [5]. However, there is limited data to show whether this reduction in error impacts function or durability after UKA. Compared to conventional methods, previous randomized controlled trials have found significant improvement in functional outcomes among high-activity patients at 1 year after UKA performed with robotic techniques.. The purpose of this study was to evaluate early functional recovery and pain levels among patients undergoing robotic-assisted or conventional UKA.

\section{Materials and Methods}

This retrospective cohort study (level III) was conducted by reviewing the records of UKA cases operated on by a single physician at multiple facilities from January 2015 to March 2018. Patients received either conventional UKA $(n=65)$ or robotic-assisted UKA $(n=96)$ depending on geographic location of the procedure and access to robotic technology at one center or another. Patient reported outcomes (PROs) were collected preoperatively, 1 week, 6 weeks, and 12 weeks postoperatively. Daily pain scores $(0-10)$ were also collected up to 4 weeks preoperatively and 12 weeks postoperatively. A linear mixed effects model was used to assess differential change over time between the two groups. Ongoing study will compare New Knee Society Scores one year after UKA.

\section{Results}

At baseline, there was no difference ( $p>0.05$ ) in average age, body mass index (BMI), Knee Injury and Osteoarthritis Outcome Scores Jr. (KOOS Jr.), or Veterans RAND 12 Item Health Survey (VR/SF12) scores between conventional and robotic UKA patients. Average preoperative pain was significantly higher among conventional vs. robotic patients (6.1 vs. $5.4 ; \mathrm{p}=0.04)$. After adjusting for this baseline difference, the conventional UKA patients still had significantly higher pain levels compared to robotic-assisted procedures at postoperative week 2 ( 4.8 vs. 4.0, $\mathrm{p}=0.05)$ and week 3 (3.9 vs. 3.1, $\mathrm{p}=0.02$ ). The Lower Extremity Functional Scale (LEFS) was not assessed preoperatively; but, both groups showed significant improvement over time $(p<0.0001)$, with robotic patients having marginally better functioning overall, particularly at week 1 (29.9 vs. 23.9, $\mathrm{p}=0.06)$. Both groups showed significant improvement in KOOS Jr. scores from pre-op (52.3) to 6 weeks $(67.6 ; \mathrm{p}<0.001)$ and 12 weeks postoperatively $(69.8 ; p<0.001)$, with no differences in the rates of improvement $(p=.69)$ between the robotic and conventional cases. Similarly, there was no difference in the mental component scores (MCS) of the VR/SF-12. However, when comparing the physical component scores (PCS) the groups had significantly different rates of improvement (time* group $p=0.04$ ). Specifically, the roboticassisted group had significantly higher PCS compared to the conventional group at 6 postoperative weeks ( 40.6 vs. 35.6; $\mathrm{p}=0.02$ ). By week 12 the conventional group had caught up to the robotic-assisted group. There were more radiographic outliers in the conventional than robotic groups. Results are summarized in Table 1. 


\section{Conclusion}

Results show that UKA performed with robotic assistance results in a more rapid recovery and less radiographic outliers with less early postoperative pain than those performed with conventional methods. Similarly, Blyth et al.[7] found significantly (55.4\%) lower VAS pain levels from postoperative day 1 through 8 weeks. LEFS scores also showed a significant improvement at 1 week postoperative in subjects with robotic assistance compared to traditional UKA. Bedard et al. [8] examined the trends and risk factors for opioid use after UKA. By decreasing postoperative pain, robotic-assisted UKA may be beneficial in reducing opioid consumption postoperatively. When comparing long term outcomes, Gilmour et al. [6] found that there were no differences between the robotic and conventional at 2 years, in terms of PROMs or any clinically significant outcome measure when analyzing 112 subjects. Further follow-up is needed to assess long term functional outcomes, as well as clinical outcomes and opioid consumption with a larger cohort of subjects. 


\section{Tables and Figures}

Table 1.

\begin{tabular}{|c|c|c|c|}
\hline & $\begin{array}{c}\text { Conventional UKA } \\
{[\mathrm{n}=65]}\end{array}$ & $\begin{array}{c}\text { Robotic-Assisted UKA } \\
{[\mathrm{n}=96]}\end{array}$ & $\mathrm{p}$-value \\
\hline Age & 60.2 & 61.6 & $\mathrm{p}=0.42$ \\
\hline Gender* & $50.8 \%$ & $54.2 \%$ & $\mathrm{p}=0.67$ \\
\hline BMI & 28.4 & 28.4 & $\mathrm{p}=0.98$ \\
\hline \multicolumn{4}{|l|}{ Pain** } \\
\hline Preop & 6.1 & 5.4 & $p=0.04$ \\
\hline Week 1 & 6.0 & 5.4 & $\mathrm{p}=0.10$ \\
\hline Week 2 & 4.8 & 4.0 & $\mathrm{p}=0.05$ \\
\hline Week 3 & 3.9 & 3.1 & $p=0.02$ \\
\hline \multicolumn{4}{|l|}{ LEFS } \\
\hline Preop & NA & NA & NA \\
\hline Week 1 & 23.9 & 29.9 & $\mathrm{p}=0.01$ \\
\hline Week 6 & 51.3 & 55.5 & $\mathrm{p}=0.13$ \\
\hline Week 12 & 56.6 & 58.2 & $\mathrm{p}=0.58$ \\
\hline \multicolumn{4}{|l|}{ KOOS Jr. } \\
\hline Preop & 50.6 & 54.3 & $\mathrm{p}=0.13$ \\
\hline Week 6 & 65.5 & 69.7 & $\mathrm{p}=0.06$ \\
\hline Week 12 & 68.6 & 70.9 & $\mathrm{p}=0.36$ \\
\hline \multicolumn{4}{|l|}{ VR/SF-12 (MCS) } \\
\hline Preop & 56.9 & 57.1 & $\mathrm{p}=0.88$ \\
\hline Week 6 & 54.4 & 55.1 & $\mathrm{p}=0.69$ \\
\hline Week 12 & 57.4 & 56.7 & $\mathrm{p}=0.73$ \\
\hline \multicolumn{4}{|l|}{ VR/SF-12 (PCS) } \\
\hline Preop & 36.4 & 36.3 & $\mathrm{p}=0.99$ \\
\hline Week 6 & 35.6 & 40.6 & $\mathrm{p}=0.02$ \\
\hline Week 12 & 45.4 & 45.4 & $\mathrm{p}=0.98$ \\
\hline
\end{tabular}

Mean Age, BMI, KOOS Jr., LEFS, and VR/SF-12 reported; with post-hoc t-test for significance

*\% Male

** Means adjusted for group baseline differences

Table 1: Conventional versus Robotic-Assisted UKA Outcome Results 
Figure 1: Average LEFS and KOOS Jr. Scores

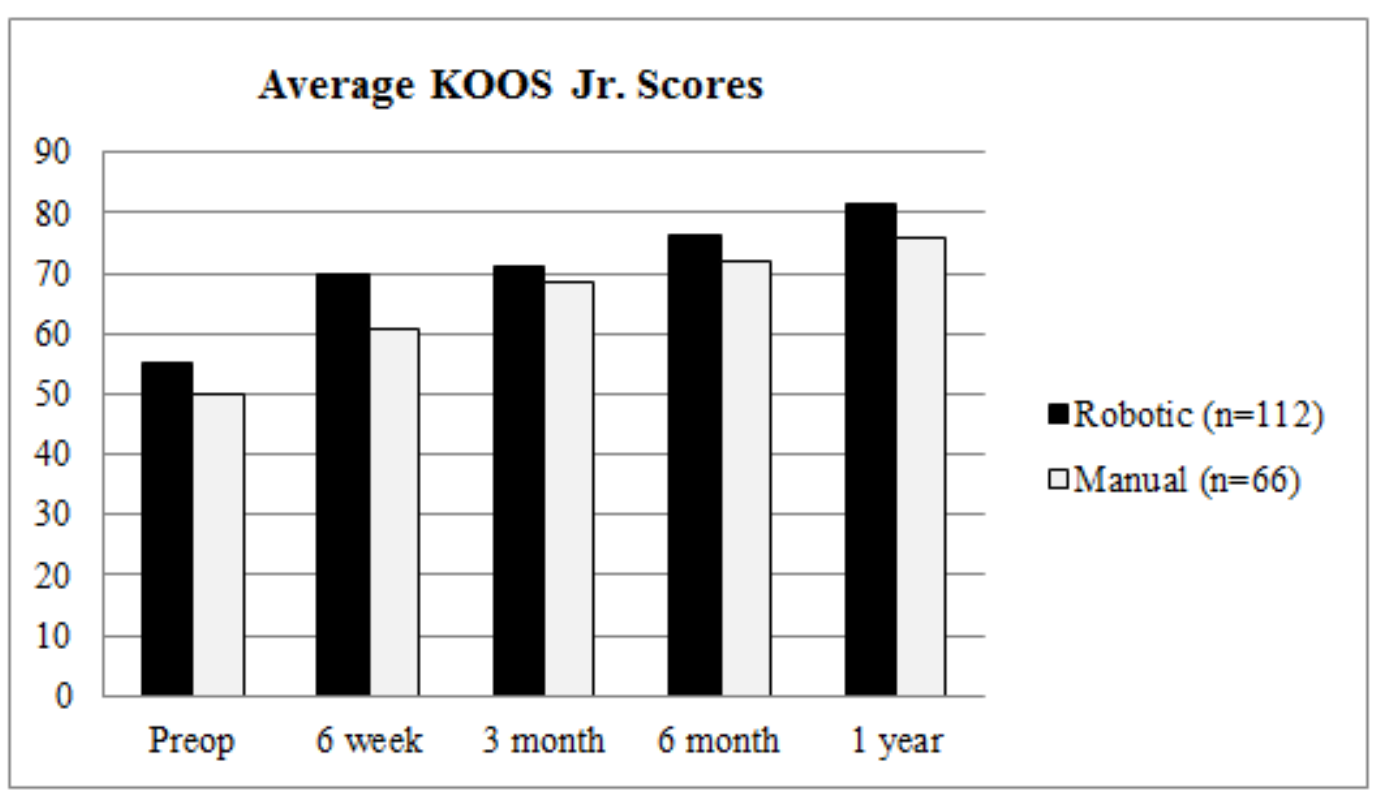

\section{Average Lower Extremity Functional Scores}

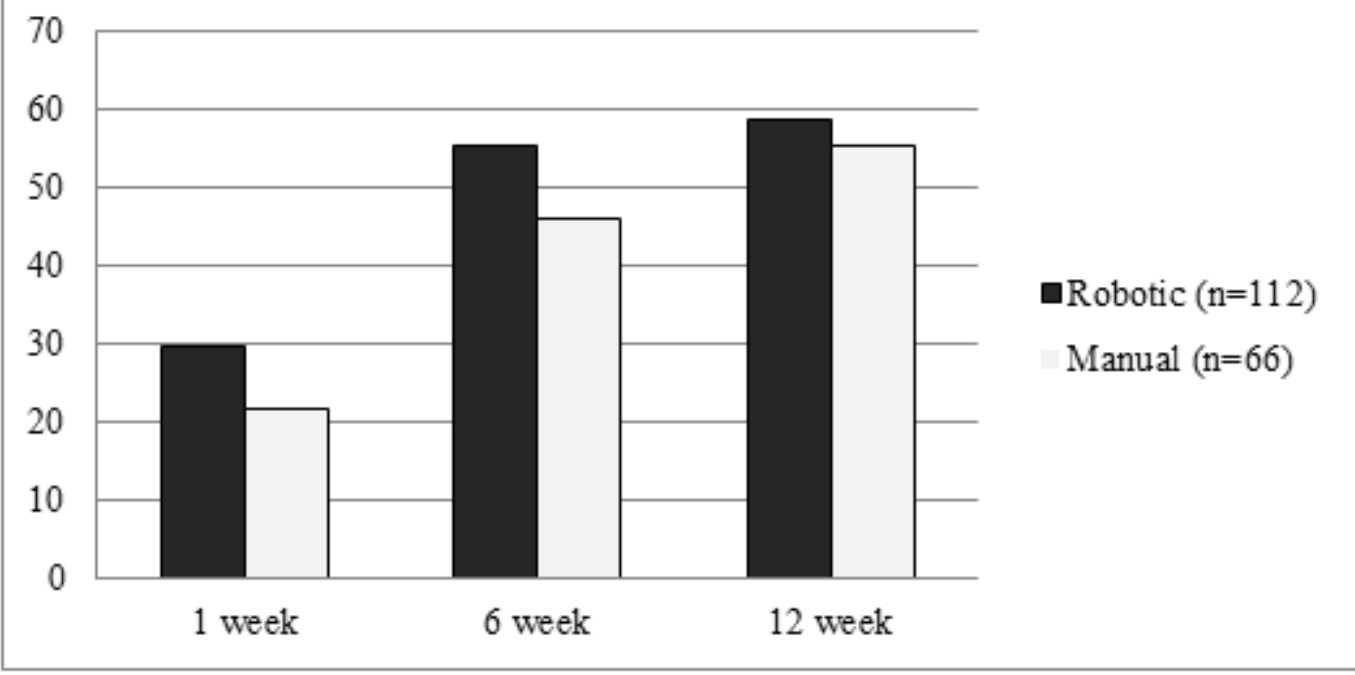




\section{References}

[1] Burn E, Sanchez-Santos MT, Pandit HG, Hamilton TW, Liddle AD, Murray DW, PinedoVillanueva R. Ten-year patient-reported outcomes following total and minimally invasive unicompartmental knee arthroplasty: a propensity score-matched cohort analysis. Knee Surg Sports Traumatol Arthrosc. 2016 May; 26(5): 1455-1464

[2] Dahm DL, Al-Rayashi W, Dajani K, Shah JP, Levy BA, Stuart MJ. Patellofemoral arthroplasty versus total knee arthroplasty in patients with isolated patellofemoral osteoarthritis. American Journal of Orthopedics. 2010 Oct;39(10):487-491.

[3] Kazarian GS, Tarity TD, Hansen EN, Cai J, Lonner JH. Significant Functional Improvement at 2 Years After Isolated Patellofemoral Arthroplasty With an Onlay Trochlear Implant, But Low Mental Health Scores Predispose to Dissatisfaction. J Arthroplasty. 2016 31:389-394

[4] Conditt MA, Bargar WL, Cobb JP, Dorr LD, Lonner JH. Current concepts in robotics for the treatment of joint disease. Hindawi Publishing Corporation; 2013.

[5] Lonner JH, Fillingham YA. Pros and Cons: A Balanced View of Robotics in Knee Arthroplasty. J Arthroplasty. 2018 Mar 30. doi: 10.1016/j.arth.2018.03.056. [Epub ahead of print]

[6] Gilmour A, MacLean AD, Rowe PJ, Banger MS, Donnelly I, Jones BG, Blyth MJG. Robotic-Arm-Assisted vs Conventional Unicompartmental Knee Arthroplasty. The 2-year Clinical Outcomes of a Randomized Controlled Trial. J Arthroplasty. 2018 Feb 21. pii: S0883-5403(18)30198-0. doi: 10.1016/j.arth.2018.02.050. [Epub ahead of print]

[7] Blyth M.J, Jones B, MacLean A, Rowe P. Two-year results of a randomized trial of robotic surgical assistance vs manual unicompartmental knee arthroplasty. Annual Meeting of the American Association of Hip and Knee Surgeons. November 2017; Dallas, TX.

[8] Bedard, N A, Demik, D E, Dowdle, S B, Callaghan, J J. Trends and risk factors for prolonged opioid use after unicompartmental knee arthroplasty. The bone \& joint journal. 2018;100-B(1 Supple A):62-67. doi:10.1302/0301-620X.100B1.BJJ-2017-0547.R1 